\title{
Characteristics and origins of non-functional Pm21 alleles in Dasypyrum villosum and wheat genetic stocks
}

Shanying $\mathrm{Zhu}^{1,2}$, Huagang $\mathrm{He}^{1 *}$

${ }^{1}$ School of Food and Biological Engineering, Jiangsu University, Zhenjiang, Jiangsu 212013, China;

${ }^{2}$ School of Environment, Jiangsu University, Zhenjiang, Jiangsu 212013, China;

Dr. Huagang He

School of Food and Biological Engineering, Jiangsu University, Zhenjiang 212013, China

Tel: $+86-511-88780201$

Fax: +86-511-88780201

E-mail: hghe@mail.ujs.edu.cn

\section{Abstract}

Most Dasypyrum villosum resources are highly resistant to wheat powdery mildew that carries Pm21 alleles. However, in the previous studies, four D. villosum lines (DvSus-1 DvSus-4) and two wheat-D. villosum addition lines (DA6V\#1 and DA6V\#3) were reported to be susceptible to powdery mildew. In the present study, the characteristics of non-functional Pm21 alleles in the above resources were analyzed after Sanger sequencing. The results showed that loss-of-functions of Pm21 alleles Pm21-NF1 Pm21-NF3 isolated from DvSus-1, DvSus-2/DvSus-3 and DvSus-4 were caused by two potential point mutations, a 1-bp deletion and a 1281-bp insertion, respectively. The non-functional Pm21 alleles in DA6V\#1 and DA6V\#3 were same to that in DvSus-4 and DvSus-2/DvSus-3, respectively, indicating that the susceptibilities of the two wheat genetic stocks came from their $D$. villosum donors. The origins of non-functional Pm21 alleles were also investigated in this study. Except the target variants involved, the sequences of Pm21-NF2 and Pm21-NF3 were identical to that of Pm21-F2 and Pm21-F3 in the resistant D. villosum lines DvRes-2 and DvRes-3, derived from the accessions GRA961 and GRA1114, respectively. It was suggested that the non-functional alleles Pm21-NF2 and Pm21-NF3 originated from the wild-type alleles Pm21-F2 and Pm21-F3. In summary, this study gives an insight into the sequence characteristics of non-functional Pm21 alleles and their origins in natural population of $D$. villosum.

Key words: Pm21; non-functional alleles; molecular tracing; origin; Dasypyrum villosum 


\section{Introduction}

Dasypyrum villosum Candargy $(2 n=2 x=14, \mathrm{VV})$ is a diploid species native to the Mediterranean region. D. villosum not only has good tiller ability and high grain protein content, but also provides tolerance to drought and cold stresses, and resistance to multiple wheat diseases, such as powdery mildew, rusts, eyespot, Take-All, and wheat spindle streak mosaic disease (De Pace et al. 2011; Wang et al. 2017). Hence, as a wild relative, D. villosum has been concerned to be an important resource for improvement of common wheat (Triticum aestivum L., $2 n=6 x=42$, AABBDD).

In 1995, a wheat-D. villosum translocation line T6AL.6VS (6V\#2S) was developed from an amphidiploid of durum wheat and D. villosum accession provided by Cambridge Botanical Garden, United Kingdom. The line T6AL.6VS carries the powdery mildew resistance gene Pm21 that confers highly resistant to all tested races of Blumeria graminis f. sp. tritici (Bgt) (Chen et al. 1995). Since then, more than 20 varieties carrying Pm21 have been developed and applied. Especially, in the middle and lower reaches of the Yangtze River Valley, the most rampant area of powdery mildew in China, wheat varieties carrying Pm21 are been planted more widely than the ones carrying Pm2a or Pm4a that are gradually losing their resistance in this region (Bie et al. 2015; He et al. 2017).

Besides 6V\#2S carrying Pm21, other wheat genetic stocks involved in chromosome 6V or 6VS of $D$. villosum have been developed by different research groups (Sears 1953; Hyde 1953; Chen et al. 1995; Li et al. 2005; Liu et al. 2011; Lukaszewski and Cowger 2017). Interestingly, among them, the addition lines DA6V\#1 (Sears 1953) and DA6V\#3 (Lukaszewski, unpublished) were reported to be susceptible to wheat powdery mildew (Qi et al. 1998; Liu et al. 2011). It is difficult to understand why these materials are susceptible since no any susceptible $D$. villosum donor was found in the previous study (Qi et al. 1998).

Recently, four seedling-susceptible D. villosum lines (DvSus-1 DvSus-4) were screened from 110 accessions in our study. Based on the finding of susceptible D. villosum resources, a fine genetic map of Pm21 was constructed, and then Pm21 was successfully characterized as a typical coiled-coil, nucleotide-binding site, leucine-rich repeat (CC-NBS-LRR) protein-encoding gene (He et al. 2017; He et al. 2018). Thus, one basic question arises: what are the characteristics of non-functional Pm21 alleles in the susceptible D. villosum lines and related wheat genetic stocks. Moreover, given that susceptible D. villosum resources are very rare, it is greatly interesting to clarify whether all or some of the susceptible $D$. villosum lines and wheat genetic stocks share the same mutation(s) of Pm21 alleles and 
whether we can trace the origins of non-functional Pm21 alleles in natural population of D. villosum. Therefore, in the present study, we made an attempt to answer the questions above via sequencing and analysis of Pm21 alleles.

\section{Materials and methods}

\section{Plant materials and growth conditions}

The resistant D. villosum line DvRes-1 carrying Pm21 was provided by Prof. Peidu Chen, Cytogenetics Institute of Nanjing Agricultural University (CI-NAU). Other D. villosum lines used in this study were provided by Germplasm Resources Information Network (GRIN) and Genebank Information System of the IPK Gatersleben (GBIS-IPK). The wheat-D. villosum disomic addition lines DA6V\#1 and DA6V\#3 were kindly provided by GRIN and Prof. Bernd Friebe, Wheat Genetic and Genomic Resource Center, Kansas State University (WGGRC-KSU), respectively (Table 1). The powdery mildew resistant wheat cultivated variety (cv.) Yangmai 18, a translocation line carrying Pm21, and the susceptible cv. Yangmai 9 were both developed in Yangzhou Academy of Agricultural Sciences (YAAS), Yangzhou, Jiangsu province. Plants were grown in greenhouse with LED light under long-day condition $(16 \mathrm{~h}$ light $/ 8 \mathrm{~h}$ dark) at $24^{\circ} \mathrm{C}$.

\section{Evaluation of powdery mildew resistance}

All plants of $D$. villosum and wheat at one-leaf stage were inoculated with Bgt isolate YZ01, a predominant isolate collected from Yangzhou by dusting fungal conidiospores from susceptible wheat cv. Yangmai 9 onto the leaves (He et al. 2016), and powdery mildew responses were assessed as resistant or susceptible at eight days post-inoculation.

\section{Allelic test}

The susceptible D. villosum line DvSus-1 was crossed with other susceptible ones (DvSus-2, DvSus-3 and DvSus-4), and three different $F_{1}$ hybrids (DvSus-1/DvSus-2, DvSus-1/DvSus-3 and DvSus-1/DvSus-4) were obtained. The susceptible wheat-D. villosum addition line DA6V\#1 was crossed with another susceptible addition line DA6V\#3 to generate $F_{1}$ hybrid DA6V\#1/DA6V\#3. Powdery mildew responses of $\mathrm{F}_{1}$ plants were assessed at eight days post-inoculation.

\section{Isolation of Pm21 alleles}

Genomic DNA was extracted from fresh leaves of seedlings by the CTAB method (Murray and Thompson 1980). PCR amplification was performed in Peltier thermal cycler (Bio-Rad, USA) in $50 \mu 1$ volume containing $1 \times$ LA PCR buffer, $0.4 \mathrm{mM}$ of each dNTP, $2 \mu \mathrm{M}$ of each primer (forward primer: 
5'-CTCTCTTCGTTACATAATGTAGTGCCT-3'), 1 unit of LA Taq DNA polymerase (TaKaRa, Japan), and $100 \mathrm{ng}$ genomic DNA. PCR was carried out with an initial denaturation at $94^{\circ} \mathrm{C}$ for $3 \mathrm{~min}, 35$ cycles of $20 \mathrm{~s}$ at $94^{\circ} \mathrm{C}, 30 \mathrm{~s}$ at $60^{\circ} \mathrm{C}, 3 \mathrm{~min}$ at $68^{\circ} \mathrm{C}$, and a final extension for $5 \mathrm{~min}$ at $72^{\circ} \mathrm{C}$. PCR products were cloned into the vector pMD18-T (TaKaRa, Japan), and then sequenced by the Sanger dideoxy sequencing method. To obtain exact sequences, each allele was isolated by three independent PCR, followed by sequencing. Full-length cDNA of each allele was further confirmed by sequencing of RT-PCR products. A phylogenetic tree was constructed by the neighbor-joining method in MEGA4 software (Tamura et al. 2007). Multiple sequence alignment analysis was performed by the CLUSTAL W tool (Thompson et al. 1994), and displayed using the GeneDoc tool (http://www.softpedia.com/get/Science-CAD/GeneDoc.shtml).

\section{Quantitative RT-PCR (qPCR) analysis}

Total RNA was isolated from leaves of the seedlings with or without Bgt inoculation using TRIzol reagent (Life Technologies, USA). The first-strand cDNA was then synthesized from $2 \mu \mathrm{g}$ of total RNA using a PrimeScript ${ }^{\mathrm{TM}}$ II 1st Strand cDNA Synthesis Kit (TaKaRa, Japan). qPCR was performed in an ABI 7300 Real Time PCR System (Life Technologies, USA) as previously described (He et al. 2018). A pair of universal primers (forward primer: 5'-TGAGTCTTCTAAACATCATTGC-3'; reverse primer: 5'-CACAACATGAACCTCGTCGT-3') was used for detection of Pm21 alleles. The wheat actin gene TaACT was used as the reference gene as reported (Bahrini et al. 2011). All reactions were run in three technical replicates for each cDNA sample.

\section{Results}

\section{Powdery mildew responses of DvSus-1 DvSus-4, DA6V\#1 and DA6V\#3}

Powdery mildew responses of four D. villosum lines (DvSus-1 $\sim$ DvSus-4) and two wheat-D. villosum addition lines (DA6V\#1 and DA6V\#3) were assessed at one-leaf stage with Bgt isolation YZ01. The results showed that all plants tested were susceptible (Fig. 1), which was in accordance with previous reports (He et al. 2017; Qi et al. 1998; Liu et al. 2011).

\section{Allelic test of susceptible factors in $D$. villosum and wheat background}

To detect whether the susceptible factors are allelic, powdery mildew responses of $F_{1}$ plants of four different crosses, including DvSus-1/DvSus-2, DvSus-1/DvSus-3, DvSus-1/DvSus-4, and DA6V\#1/DA6V\#3, were detected. The results demonstrated that all the $\mathrm{F}_{1}$ hybrids were susceptible to 
powdery mildew (Fig. 1), indicating no allelic complementation observed. Therefore, loss-of-function mutations in the susceptible D. villosum lines and wheat-D. villosum addition lines occurred in Pm21 alleles.

\section{Quantitative RT-PCR (qPCR) analysis of Pm21 alleles}

To detect the relative transcription levels of Pm21 alleles in different materials, qPCR was carried out. The data showed that Pm21 alleles were all constitutively transcribed at relatively equal levels and displayed similar responses to Bgt, compared with the resistant D. villosum line DvRes-1 (Fig. 2). It was indicated that loss-of-functions of Pm21 alleles might be caused by mutations in their encoding sequences rather than by the difference in their transcription patterns.

\section{Sequence characteristics of non-functional Pm21 alleles}

To reveal the sequence characteristics, non-functional alleles of Pm21 were isolated from the four susceptible $D$. villosum lines. The result showed that the genomic sequence of non-functional allele Pm21-NF1 in DvSus-1 was 3,699 bp. The corresponding open reading frame (ORF) was 2,730 bp that had 98 single nucleotide polymorphisms (SNPs) contrasted to Pm21. However, Pm21-NF1 only had two specific variation sites in contrast to 38 non-redundant alleles isolated from resistant D. villosum accessions reported recently (He et al. 2018). The first one was a G-to-T transversion at the position 61 that leaded to an amino acid change (A21S) in the CC domain. The second one was a A-to-G transition at the position 821 that caused another amino acid change (D274G), the second aspartate (D) in kinase-2 motif (consensus sequence: LLVLDDVW) of the NBS domain (Meyers et al. 1999) (Fig. 3A). Further analysis showed that the second D was conserved in all the 19 disease resistance proteins characterized from wheat, barley and Arabidopsis (Fig. 4).

The genomic sequence of non-functional allele Pm21-NF2 in DvSus-2 was 3,698 bp. The ORF of Pm21-NF2 had a 1-bp (A) deletion after the position 876 that leaded to three tandem premature stop codons (TAA-TAG-TAA) in the NBS-encoding region and formed a truncated protein with 296 aa in length (Fig. 3B). The sequence of Pm21 allele in DvSus-3 was identical to that in DvSus-2, which indicated the same origin.

The non-functional allele Pm21-NF3 in the susceptible D. villosum line DvSus-4 were 4,988 bp in length. Pm21-NF3 contained a 1281-bp insertion that is a kind of repeat sequence present in the genome of wheat cv. Chinese Spring (Fig. 3C). The insertion in Pm21-NF3 introduced a premature stop codon, which leaded to a truncated LRR domain. 
Pm21 alleles in wheat-D. villosum addition lines DA6V\#1 and DA6V\#3 were also cloned and sequenced in this study. Interestingly, the non-functional Pm21 alleles in DA6V\#1 and DA6V\#3 were identical to that in DvSus-4 and DvSus-2/DvSus-3, respectively (Fig. 3B and Fig. 3C). It was indicated that the non-functional Pm21 alleles in the two wheat genetic stocks were both derived from their $D$. villosum donors.

\section{Molecular tracing of the origins of non-functional Pm21 alleles}

To trace the origins of non-functional Pm21 alleles, a neighbor-joining phylogenetic tree was constructed based on the sequences of Pm21 alleles (Fig. 5). The data showed that DvSus1, DvSus2, DvSus-3, DvRes-2 and GRA1164 were clustered in a clade. In contrast to the alleles in DvRes-2 and GRA1164, the non-functional allele Pm21-NF1 in DvSus1 had eight and ten SNPs, and Pm21-NF2 in DvSus2/DvSus-3 had one and three SNPs, respectively. It was indicated that the non-functional allele Pm21-NF2 in DvSus2/DvSus-3 might originate from the corresponding functional allele Pm21-F2 in DvRes-2 that was derived from the accession GRA961 (Fig. 6), whereas the origin of Pm21-NF1 in DvSus 1 could not be traced yet in the accessions tested.

The phylogenetic tree also showed that DvSus-4, DvRes-3 and GRA1113 were clustered in an independent clade (Fig. 5). Sequence analysis demonstrated that, except the insertion sequence, Pm21-NF2 in DvSus-4 had five SNPs contrasted to that in GRA1113, but no difference with that in

DvRes-3. It was suggested that the non-functional allele Pm21-NF3 in DvSus4 might originate from the corresponding functional allele Pm21-F3 in DvRes-3 that was derived from the accession GRA1114 (Fig. 6).

\section{Discussion}

Pm21, originating from $D$. villosum, confers highly effective resistance to wheat powdery mildew at the whole growth stages. To genetic map Pm21, 46 D. villosum accessions were investigated to find susceptible resource; however, none was obtained (Qi et al. 1998). Hence, since 1998, researchers believe that all D. villosum resources are resistant to powdery mildew. Recently, four seedling-susceptible D. villosum lines (DvSus-1 $\sim$ DvSus-4) showing complex resistance associated with growth stage were identified from different natural populations, which allows to fine map and clone Pm21 (He et al. 2017; He et al. 2018). In this study, the characteristics of Pm21 alleles in these susceptible lines were further investigated by Sanger sequencing. The data demonstrated that the variation of Pm21 alleles in the four seedling-susceptible D. villosum lines involved in point mutation, 
deletion and insertion, respectively.

DvSus-2 and DvSus-3 contained the same allele Pm21-NF2 that involved in a 1-bp deletion and leaded to a truncated PM21 protein. The length of the truncated protein (296 aa) is similar to that in the susceptible mutant lines Y18-S7 (281 aa) and Y18-S21 (309 aa) derived from EMS-induced wheat cv. Yangmai 18 carrying Pm21. In DvSus-4, the non-functional allele Pm21-NF3 had a 1281-bp insertion of repeat sequence that leaded to lose the last four LRR motifs (13th to 16th). LRR domain of disease resistance protein plays an important role in perception of pathogen effector. Recent observation on three susceptible mutants of Yangmai 18 (Y18-S8, Y18-S30 and Y18-S47) showed that each mutation in the 14th LRR motifs, leading to premature stop codon or amino acid change, can impair the Pm21 resistance (He et al. 2018). It was suggested that an important site(s) for perception of Bgt effector is lost in the truncated protein encoded by Pm21-NF3. Taken together, it is supported that both Pm21-NF3 and Pm21-NF3 were lost the resistance to powdery mildew.

Allelic tests on the crosses, DvSus-1/DvSus-2, DvSus-1/DvSus-3 and DvSus-1/DvSus-4, suggested that all mutations leading to lose resistance occurred in the alleles. Therefore, it was proposed that the allele Pm21-NF1 in DvSus-1 was also non-functional. Contrasted to Pm21, Pm21-NF1 had a number of SNPs; however, only two (G61T and A821G) were specific in all tested Pm21 alleles that cause amino acid changes (A21S and D274G). A21S lied in the CC domain; however, whether it could impair the Pm21 resistance remains unclear. D274 was the second aspartate (D) of kinase-2 motif (also called Walker B motif; consensus sequence: LLVLDDVW) in the NBS domain that is considered to act as the catalytic base for ATP hydrolysis and activation of disease resistance protein (Meyers et al. 1999; Tameling et al. 2006). Here, we investigated 19 plant disease resistance proteins and found that the second D of kinase-2 motif was highly conserved. This result supported that the amino acid change D274G might lead to loss-of-function of Pm21-NF1.

In this research, the origins of Pm21-NF2 and Pm21-NF3 were also successfully traced by sequence analysis. Except the mutation sites, Pm21-NF2 and Pm21-NF3 were identical to Pm21-F2 and Pm21-F3 isolated from the resistant D. villosum lines DvRes-2 and DvRes-3, derived from the accessions GRA961 and GRA1114, respectively. Therefore, it was suggested that Pm21-F2 and Pm21-F3 are the wild-type alleles corresponding to Pm21-NF2 and Pm21-NF3, respectively.

Previously, researchers observed that wheat-D. villosum addition lines DA6V\#1 and DA6V\#3 were susceptible to powdery mildew (Qi et al. 1998; Liu et al. 2011). Due to the utilization of colchicine, an 
effectively chemical mutagen (Gilbert and Patterson 1965), in the incorporation of alien genome, it is difficult to explain whether the susceptibilities of DA6V\#1 and DA6V\#3 came from colchicine treatment or alien donors. In the present study, via allele sequencing, we revealed that Pm21 alleles in DA6V\#1 and DA6V\#3 were identical to Pm21-NF3 and Pm21-NF2, respectively. These results suggested that both of the variations of Pm21 alleles in DA6V\#1 and DA6V\#3 originated from their D. villosum donors.

\section{References}

Bahrini I, Ogawa T, Kobayashi F, Kawahigashi H, Handa H (2011) Overexpression of the pathogen-inducible wheat TaWRKY45 gene confers disease resistance to multiple fungi in transgenic wheat plants. Breed Sci 61(4):319-326

Bie T, Zhao R, Zhu S, Chen S, Cen B, Zhang B, Gao D, Jiang Z, Chen T, Wang L, Wu R, He H (2015) Development and characterization of marker $M B H 1$ simultaneously tagging genes $P m 21$ and $P m V$ conferring resistance to powdery mildew in wheat. Mol. Breeding 35:189

Chen PD, Qi LL, Zhou B, Zhang SZ, Liu DJ (1995) Development and molecular cytogenetic analysis of wheat-Haynaldia villosa 6VS/6AL translocation lines specifying resistance to powdery mildew. Theor Appl Genet 91:1125-1128

De Pace C, Vaccino P, Cionini PG, Pasquini M, Bizzarri M, Qualset CO (2011) Dasypyrum. Chapter 4. In: Kole C (ed) Wild crop relatives: genomic and breeding resources, Cereals, Springer, Berlin, pp185-292

Gilbert SK, Patterson FL (1965) Colchicine-induced mutants in Decatur barley. Crop Sci 5: 44-47

He H, Ji Y, Zhu S, Li B, Zhao R, Jiang Z, Bie T (2017) Genetic, physical and comparative mapping of the powdery mildew resistance gene Pm21 originating from Dasypyrum villosum. Front Plant Sci 2017, 8:1914

He H, Zhu S, Zhao R, Jiang Z, Ji Y, Ji J, Qiu D, Li H, Bie T (2018) Pm21, encoding a typical CC-NBS-LRR protein, confers broad-spectrum resistance to wheat powdery mildew disease. Mol Plant (https://doi.org/10.1016/j.molp.2018.03.004)

Hyde BB (1953) Addition of individual Haynaldia villosa chromosomes to hexaploid wheat. Am J Bot 40:174-182

Li H, Chen X, Xin ZY, Ma YZ, Xu HJ, Chen XY, Jia X (2005) Development and identification of wheat-Haynaldia villosa T6DL.6VS chromosome translocation lines conferring resistance to powdery 
mildew. Plant Breed 124:203-205

Liu C, Qi L, Liu W, Zhao W, Wilson J, Friebe B, Gill BS (2011) Development of a set of compensating Triticum aestivum - Dasypyrum villosum Robertsonian translocation lines. Genome 54:1-9

Lukaszewski AJ, Cowger C (2017) Re-engineering of the Pm21 transfer from Haynaldia villosa to bread wheat by induced homoeologous recombination. Crop Sci 57:2590-2594

Meyers BC, Dickerman AW, Michelmore RW, Sivaramakrishnan S, Sobral BW, Young ND (1999) Plant disease resistance genes encode members of an ancient and diverse protein family within the nucleotide-binding superfamily. Plant J 20:317-332

Murray MG, Thompson YF (1980) Rapid isolation of high molecular weight plant DNA. Nucleic Acids Res 8:4321-4325

Qi LL, Wang SL, Chen PD, Liu DJ, Gill BS (1998) Identification and physical mapping of three Haynaldia villosa chromosome-6V deletion lines. Theor Appl Genet 97:1042-1046

Sears ER (1953) Addition of the genome of Haynaldia villosa to Triticum aestivum. Am J Bot 40:168-174

Tameling WIL, Vossen JH, Albrecht M, Lengauer T, Berden JA, Haring MA, Cornelissen BJC, Takken FLW (2006) Mutations in the NB-ARC domain of I-2 that impair ATP hydrolysis cause autoactivation. Plant Physiol 140:1233-1245

Tamura K, Dudley J, Nei M, Kumar S (2007) MEGA4: Molecular Evolutionary Genetics Analysis (MEGA) software version 4.0. Mol Biol Evol 24:1596-1599

Thompson J, Higgins D, Gibson T (1994) CLUSTAL W: improving the sensitivity of progressive multiple sequence alignment through sequence weighting position specific gap penalties and weight matrix choice. Nucleic Acids Res 22:4673-4680

Wang H, Dai K, Xiao J, Yuan C, Zhao R, Doležel J, Wu Y, Cao A, Chen P, Zhang S, Wang X (2017) Development of intron targeting (IT) markers specific for chromosome arm 4VS of Haynaldia villosa by chromosome sorting and next-generation sequencing. BMC genomics 18:167 
Table 1 D. villosum lines and wheat genetic stocks used in this study. CI-NAU: Cytogenetics Institute, Nanjing Agricultural University. GBIS-IPK: Genebank Information System of the IPK Gatersleben. GRIN: Germplasm Resources Information Network. WGGRC-KSU: Wheat Genetic and Genomic Resource Center, Kansas State University

\begin{tabular}{|c|c|c|c|c|c|}
\hline Line & Allele $^{\mathrm{a}}$ & $\begin{array}{l}\text { Powdery } \\
\text { mildew } \\
\text { response }\end{array}$ & Origin & $\begin{array}{l}\text { Original } \\
\text { accession }\end{array}$ & Provider \\
\hline DvRes-1 & $\operatorname{Pm} 21$ & Resistant & United Kingdom & Unknown & CI-NAU \\
\hline DvRes-2 & Pm21-F2 & Resistant & Unknown & GRA 961 & GBIS-IPK \\
\hline DvRes-3 & Pm21-F3 & Resistant & Italy & GRA 1114 & GBIS-IPK \\
\hline DvSus-1 & Pm21-NF1 & Susceptible & Greece & GRA 2738 & GBIS-IPK \\
\hline DvSus-2 & Pm21-NF2 & Susceptible & Unknown & GRA 962 & GBIS-IPK \\
\hline DvSus-3 & Pm21-NF2 & Susceptible & Italy & GRA 1105 & GBIS-IPK \\
\hline DvSus-4 & Pm21-NF3 & Susceptible & Former Soviet Union & PI 598390 & GRIN \\
\hline DA6V\#1 & Pm21-NF3 & Susceptible & Developed by Sears & GSTR 315 & GRIN \\
\hline DA6V\#3 & Pm21-NF2 & Susceptible & Developed by Lukaszewski & TA5618 & WGGRC-KSU \\
\hline
\end{tabular}

a Pm21-NF1 Pm21-NF3 were non-functional alleles of Pm21 whose corresponding functional alleles were designated as Pm21-F1 Pm21-F3, respectively. In this study, Pm21-F2 and Pm21-F3 were successfully characterized, whereas Pm21-F1 was not found in D. villosum accessions tested. 


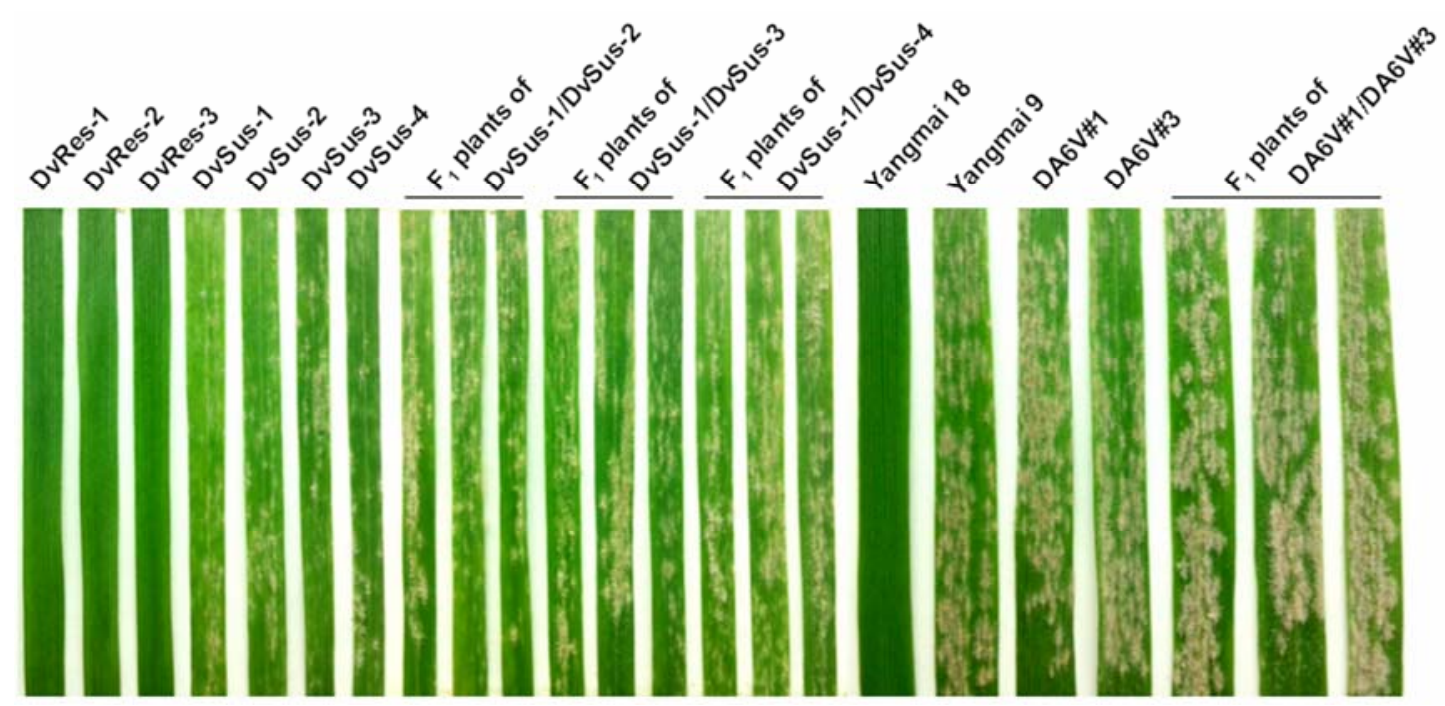

Fig. 1 Powdery mildew responses of different $D$. villosum lines, wheat genetic stocks, and $\mathrm{F}_{1}$ plants of the crosses DvSus-1/DvSus-2, DvSus-1/DvSus-3, DvSus-1/DvSus-4, Dv6V\#1/ Dv6V\#3 to Bgt isolate YZ01 at one-leaf stage.

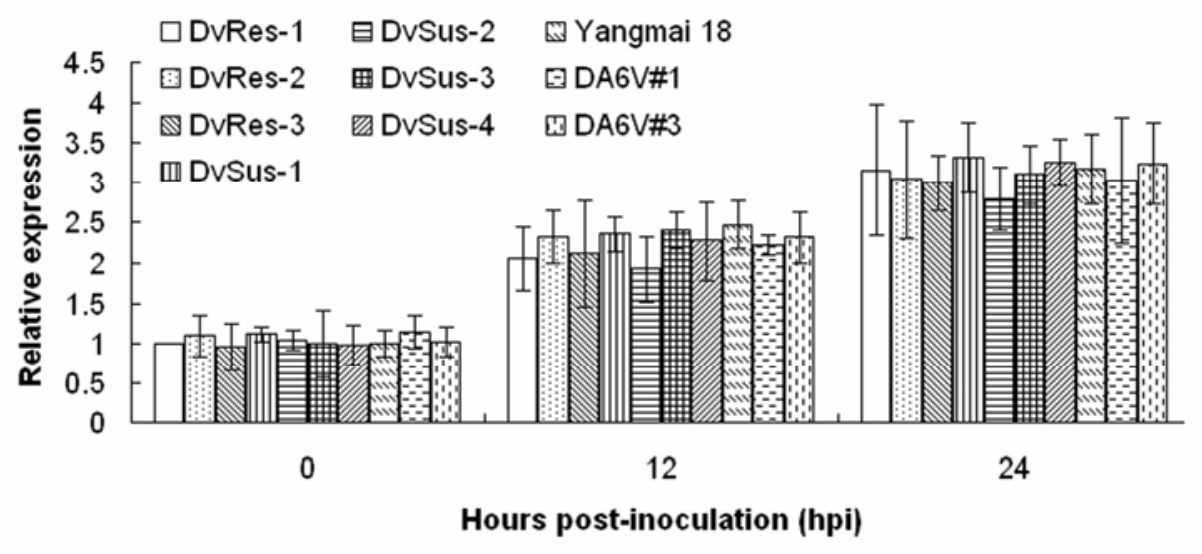

Fig. 2 Transcription patterns of Pm21 alleles in the leaves of the susceptible D. villosum lines and wheat genetic stocks inoculated with Bgt isolation YZ01, using quantitative RT-PCR (qPCR). The wheat actin (TaACT) gene was used as reference gene. The transcriptional values of Pm21 allele in the D. villosum line DvRes-1 was set to 1 at 0 hpi. The error bars indicate standard deviation (SD) from three technical replicates. 

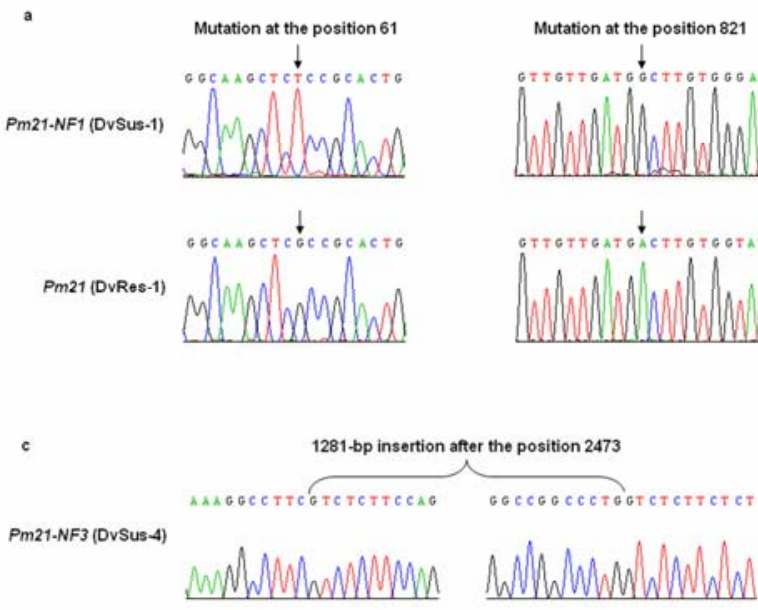

PM21-NF3 (DAGVE11
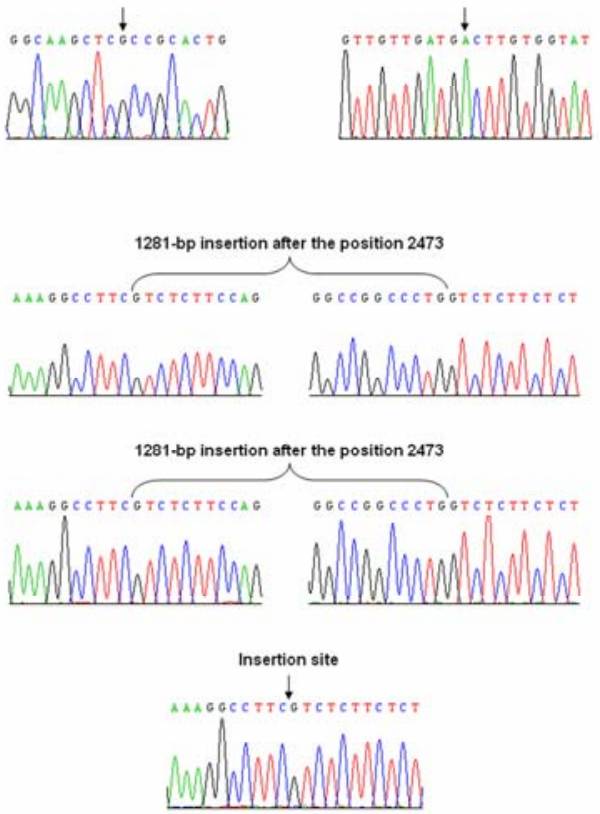

Pm21-F3 (DvRes-3)

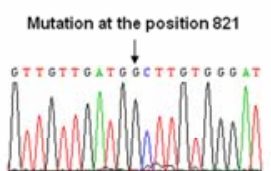
tret
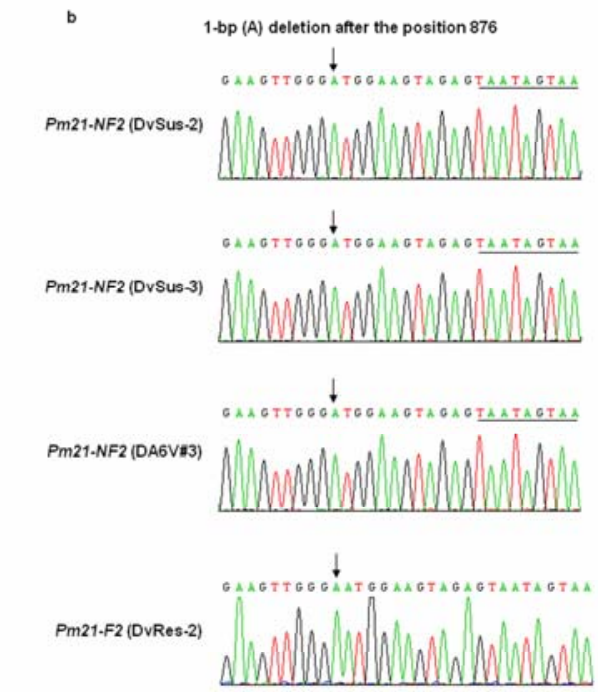

\section{西}




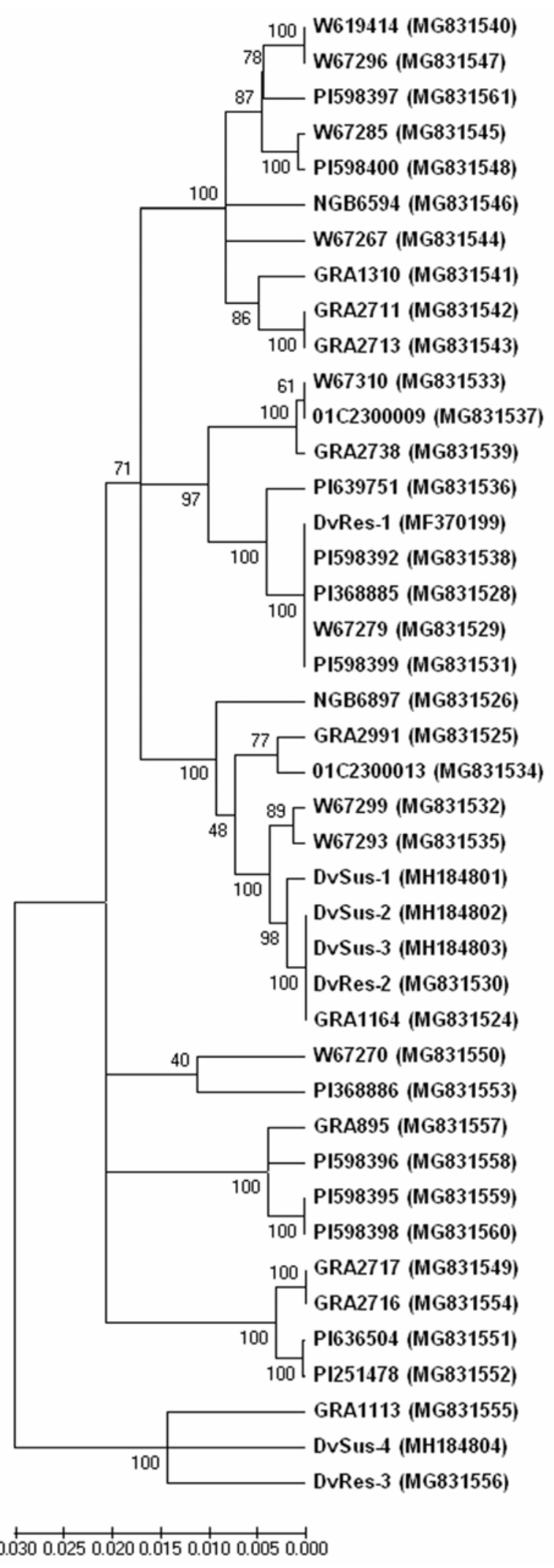

Fig. 5 Phylogenetic tree based on the sequences of non-functional Pm21 alleles obtained in this study, together with other Pm21 alleles reported in our recent work (He et al. 2018). The accession numbers of alleles are shown in brackets. 


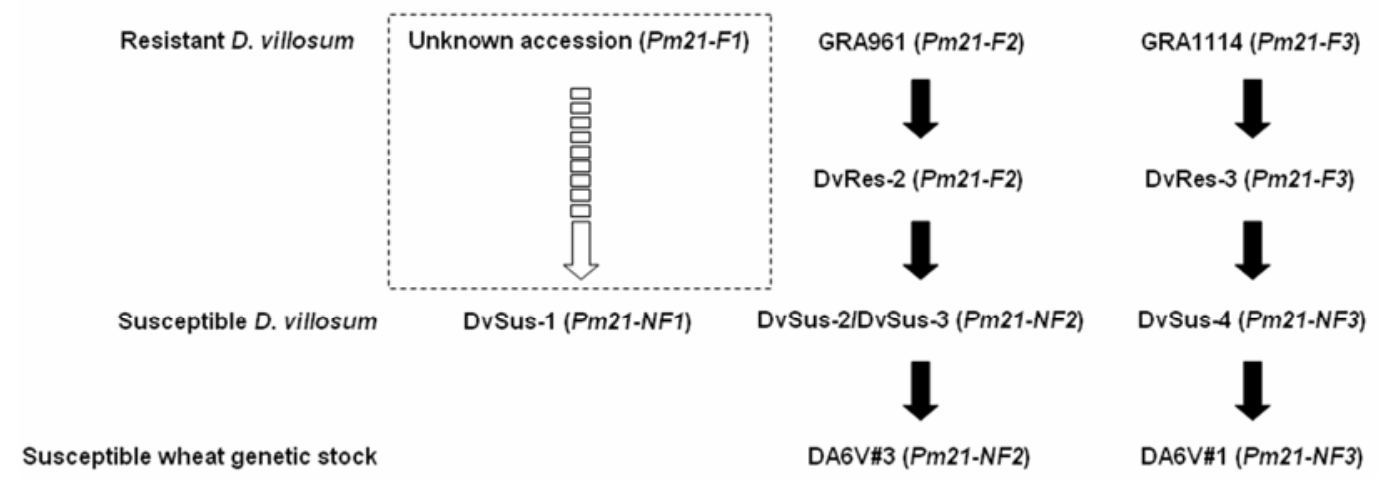

Fig. 6 Origins of the non-functional alleles Pm21-NF1 Pm21-NF3, corresponding to functional alleles Pm21-F1 Pm21-F3, respectively. Among them, Pm21-F1 was not detected in D. villosum accessions that is marked by a dashed box. 